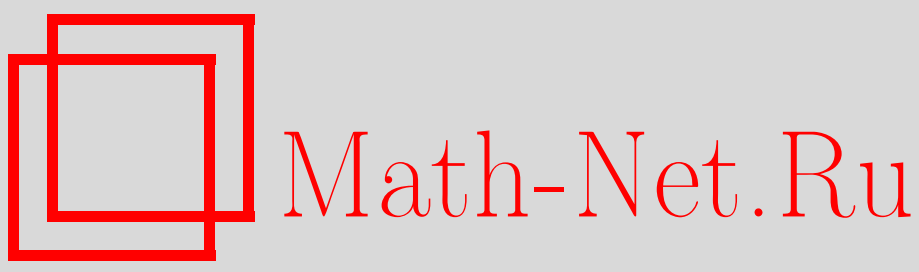

К. А. Зубрилин, Об идеале Бэра в алгебрах, удовлетворяющих тождествам Капелли, Матем. сб., 1998, том 189, номер 12, 73-82

DOI: https://doi.org/10.4213/sm375

Использование Общероссийского математического портала Math-Net.Ru подразумевает, что вы прочитали и согласны с пользовательским соглашением http://www . mathnet.ru/rus/agreement

Параметры загрузки:

IP : 3.85 .5 .30

26 апреля 2023 г., 06:34:00 
УДК 512

\author{
К.А. Зубрилин
}

\title{
Об идеале Бэра в алгебрах, удовлетворяющих тождествам Капелли
}

\begin{abstract}
Исследована структура идеала Бэра конечно порожденной алгебры произвольной конечной сигнатуры над произвольным полем или нётеровьм коммутативно-ассоциативнњм колцом, удовлетворяющей системе тождеств Капелли порядка $n+1$.

Доказано, что длина бэровской цепочки идеалов в такой алгебре не превосходит $n$. Доказано, что факторалгебра такой алгебры по наибольшему нильпотентному идеалу представима.

Библиография: 10 названий.
\end{abstract}

Для всякой алгебры $A$ сигнатуры $\Omega$ можно определить идеал Бэра $B(A)$ при помощи трансфинитной индукции. Пусть $B_{0}(A)=(0)$. Для любого ординала $\alpha$, если он непредельный, т.е. имеет вид $\alpha=\beta+1$, полагаем $B_{\alpha}(A)$ как полный прообраз суммы всех нильпотентных идеалов в $A / B_{\beta}(A)$. Если $\alpha$ пределен, то $B_{\alpha}(A)=\bigcup_{\beta<\alpha} B_{\beta}(A)$. Согласно принципу трансфиннитной индукции найдется ординал $\alpha$ такой, что $B_{\alpha}(A)=B_{\alpha+1}(A)$. Тогда идеал $B_{\alpha}(A)$ назовем идеалом Бэра алгебры $A$. Нетрудно видеть, что $B(A)$ является полупервичньм идеалом алгебры $A$ и содержится в любом другом полупервичном идеале алгебры $A$ (см. [1]). Бэровская цепочка идеалов может быть определена для алгебры произвольной сигнатуры.

Говорят, что алгебра удовлетворяет системе тождеств Капелли порядка $n$, если всякий полином, полилинейный и кососимметричный по некоторым $n$ своим переменным, является тождеством этой алгебры.

Тождества Капелли могут быть определены для произвольной сигнатуры, хотя первоначально тождества Капелли рассматривались только для ассоциативных алгебр (см. [2]).

Тождества Капелли, с одной стороны, являются естественным обобщением тождества коммутативности и тождества ассоциативности. Если бинарная алгебра удовлетворяет тождествам Капелли порядка 2, то эта алгебра коммутативна и ассоциативна. С другой стороны, выполнение тождеств Капелли может рассматриваться как обобшение свойства конечномерности алгебры, поскольку во всякой $m$-мерной алгебре выполняются тождества Капелли порядка $m+1$.

Впервые тождества Капелли были введены в рассмотрение Ю. П. Размысловым в [2] в связи с решением проблемы радикала ассоциативной PI-алгебры.

Комбинаторные аспекты тождеств Капелли произвольной сигнатуры изучались Размысловым в [3]. В этой же работе была построена структурная теория алгебр произвольной конечной сигнатуры над полем нулевой характеристики, удовлетворяющих системе тождеств Капелли некоторого порядка.

Работа выполнена при финансовой поддержке Российского фонда фундаментальных исследований (грант № 96-01-01088).

(C) К.А. Зуврилин 1998 
Развитию структурной теории алгебр произвольной конечной сигнатуры над полем произвольной характеристики или нётеровым коммутативно-ассоциативньм кольцом, удовлетворяющих системе тождеств Капелли некоторого порядка, посвящены работы [4]-[7]. В этих работах показано, что структурная теория таких алгебр является содержательной и многие результаты о структуре ассоциативных PI-алгебр и конечномерных алгебр могут быть перенесены на случай алгебр произвольной конечной сигнатуры, удовлетворяюших тождествам Капелли некоторого порядка.

В [7] доказано, что для всякой конечно порожденной алгебры $L$ произвольной конечной сигнатуры над полем произвольной характеристики или нётеровым коммутативно-ассоциативньм кольцом, удовлетворяющей тождествам Капелли некоторого порядка, сушествует наибольший нильпотентный идеал $N(L)$, т.е. идеал $B_{1}(L)$ является (наибольшим) нильпотентным идеалом.

В [5] доказано, что идеал Бэра $B(L)$ конечно порожденной алгебры $L$ произвольной конечной сигнатуры над полем произвольной характеристики или нётеровым коммутативно-ассоциативным кольцом, удовлетворяющей тождествам Капелли некоторого порядка, совпадает с $B_{s}(L)$ для некоторого $s \in\{0\} \cup \mathbb{N}$.

Структуру бэровского идеала алгебры, удовлетворяющей тождествам Капелли, описывает

TЕОРеМА 1. Пусть $L$ - конечно порожденная алгебра конечной сигнатуры $\Omega$ над полем произвольной характеристики или произвольныцм нётеровым коммутативно-ассоциативныц кольцом $C$ и $C$-алгебра L удовлетворяет тождествам Капелли порядка $n+1$. Тогда в алгебре $L$ идеал Бәра $B(L)$ совпадает с $B_{n}(L)$.

ОПРеДЕЛЕниЕ 1. Алгебра $L$ над кольцом $C$ называется представимой, если эта алгебра может быть вложена как $C$-подалгебра в $\widetilde{C}$-алгебру $\widetilde{L}$, где $\widetilde{C}$-конечно порожденная коммутативно-ассоциативная $C$-алгебра, $\widetilde{L}$ - конечно порожденный $\widetilde{C}$-модуль.

В работе [6] автором доказано, что во всякой конечно порожденной алгебре $L$ над полем произвольной характеристики или произвольным нётеровым коммутативно-ассоциативньм кольцом $C$, удовлетворяющей тождествам Капелли порядка $n+1$, найдется нильпотентньй идеал $I$ такой, что класс нильпотентности идеала $I$ не превосходит $n$ и алгебра $L / I$ представима. Ранее эта теорема была доказана Размысловым в [3] для алгебры над полем нулевой характеристики, затем автором в [5] - для алгебры над произвольным полем или нётеровым коммутативно-ассоциативным кольцом, причем в обоих случаях утверждалось, что класс нильпотентности идеала $I$ не превосходит $2^{n+1}-2$. В [3], [6] был указан способ построения идеала $I$, и этот идеал был назван препятствием к представимости.

Новым результатом о представимости является

TЕОРема 2. Пусть $L$ - конечно порожденная алгебра конечной сигнатуры $\Omega$ над полем произвольной характеристики или произвольныцм нётеровым коммутативно-ассоциативньцм кольцом $C$ и $C$-алгебра L удовлетворяет тождествам Капелли некоторого порядка. Тогда алгебра $L / N(L)$, где $N(L)$ - наибольший нильпотентный идеал алгебры L, представима.

В конечно порожденной ассоциативной PI-алгебре $L$ по теореме Брауна-Keмера-Размыслова всегда $B(L)=B_{1}(L)=N(L)$ (см. [8], [9]). В этом случае 
алгебра $L / N(L)$ полупервична, а значит, представима по теореме Капланского (см. [10]). Поэтому теоремы 1, 2 можно рассматривать как обобщения теорем Брауна-Кемера-Размыслова и Капланского, соответственно, на случай конечно порожденных алгебр конечной сигнатуры, удовлетворяюших тождествам Капелли некоторого порядка, над полем произвольной характеристики или произвольным нётеровым коммутативно-ассоциативным кольцом.

Из определения бэровской цепочки идеалов и теоремы 2 вытекает

СЛЕДСТВИЕ 1. Пусть $L$ - конечно порожденная алгебра конечной сигнатуры $\Omega$ над полем произвольной характеристики или произвольным нётеровым коммутативно-ассоциативным кольцом $C$ и C-алгебра L удовлетворяет тождествам Капелли некоторого порядка. Тогда алгебра $L / B_{q}(L)$, где $B_{q}(L)$ - әлемент с номером $q$ из бәровской чепочки идеалов алгебры $L$, представима.

При доказательстве теорем 1, 2 сушественно используются структурные теоремы и комбинаторные методы из [3]-[7].

\section{§1. Предварительные сведения и определения}

Пусть $C$ - коммутативное и ассоциативное кольцо с единицей. Будем понимать под $C$-алгеброй сигнатуры $\Omega C$-модуль $A$, на котором для каждого $\omega \in \Omega$ определена $C$-полилинейная операция с "арностью" $n=n(\omega)$

$$
\omega: \underbrace{A \otimes_{C} A \otimes_{C} \cdots \otimes_{C} A}_{n \text { раз }} \rightarrow A .
$$

Для всякой $C$-алгебры $A$ сигнатуры $\Omega$ обозначим через $D(A)$ ассоциативную $C$-алгебру, которая порождена в $\operatorname{End}_{C} A$ единичным оператором и всеми элементами вида $\left.f\left(\widehat{x}_{0}, x_{1}, \ldots, x_{q}\right)\right|_{x_{1}=v_{1}, \ldots, x_{q}=v_{q}}$, где $f\left(x_{0}, \ldots, x_{q}\right)$ - полином из абсолютно свободной $C$-алгебры сигнатуры $\Omega$, который линеен по $x_{0}$, и по определению $f\left(\widehat{x}_{0}, v_{1}, \ldots, v_{q}\right) \times v=f\left(v, v_{1}, \ldots, v_{q}\right)$ для всех $v \in A$. Если $A-$ бинарная алгебра, то алгебра $D(A)$ назьвается алгеброй левых и правых умножений, ассоциированной с алгеброй $A$.

Алгебра $A$ обладает канонической структурой левого $D(A)$-модуля. Эта структура задается естественным вложением алгебры $D(A)$ в алгебру $\operatorname{End}_{C} A$. Идеалами алгебры $A$ называют подмодули $D(A)$-модуля $A$.

Дополнительно будем предполагать, что среди сигнатурных операций алгебры $A$ имеется операция, "арность" которой $\geqslant 2$.

Будем говорить, что идеал $I$ алгебры $A$ сигнатуры $\Omega$ является произведением идеалов $I_{1}, \ldots, I_{q}$ алгебры $A$, если идеал $I$ порожден элементами вида

$$
\left.g\right|_{x_{1}=v_{1}, \ldots, x_{q}=v_{q}, \ldots, x_{r}=v_{r}},
$$

где $g\left(x_{1}, \ldots, x_{q}, \ldots, x_{r}\right)$ - полином из абсолютно свободной $C$-алгебры сигнатуры $\Omega$, полилинейньй по $x_{1}, \ldots, x_{q}$ и зависящий, быть может, от других переменных $x_{i}, q<i ; v_{i} \in I_{i}, 1 \leqslant i \leqslant q$, и $v_{i} \in A$, если $i>q$. Будем писать в этом случае, что $I=\left(I_{1} \ldots . I_{q}\right)$. Произведение идеалов, определенное таким образом, является коммутативньм. 
ОПРЕДЕЛЕНИЕ 2 . Идеал $I$ алгебры $A$ сигнатуры $\Omega$ называется нильпотентнылм класса $n$, если

$$
(\underbrace{I \ldots I I I I}_{n \text { раз }}) \neq(0), \quad(\underbrace{I \ldots I \ldots I}_{n+1 \text { раз }})=(0) .
$$

Алгебра $A$ сигнатуры $\Omega$ называется полупервичной, если алгебра $A$ не содержит нильпотентных идеалов.

Иногда вместо $(\underbrace{I \ldots \ldots I}_{n \text { раз }})$ мы будем писать $I^{n}$.

Отметим, что сумма двух или любого конечного числа нильпотентњых идеалов является нильпотентным идеалом, поскольку идеал $\sum_{i=1}^{m} I_{i}$, где $I_{i}, 1 \leqslant i \leqslant m,-$ нильпотентный идеал класса $n_{i}$ алгебры $A$ сигнатуры $\Omega$, нильпотентен в алгебре $A$ класса не вьше $\sum_{i=1}^{m} n_{i}$.

Пусть $\operatorname{Cap}_{n}(L)$ - идеал алгебры $L$, порожденньй элементами вида

$$
\left.f\right|_{x_{1}=w_{1}, \ldots, x_{n}=w_{n}, x_{n+1}=w_{n+1}, \ldots}
$$

где $f$ - произвольный полином сигнатуры $\Omega$, который полилинеен и кососимметричен по переменным $x_{1}, \ldots, x_{n}$ и, возможно, зависит от других переменных типа $x$; $w_{1}, \ldots, w_{n}, w_{n+1}, \ldots$-произвольные элементы из алгебры $L$. Из определения идеала $\operatorname{Cap}_{n}(L)$ следует, что алгебра $L / \operatorname{Cap}_{n}(L)$ удовлетворяет системе тождеств Капелли порядка $n$. В частности, $\operatorname{Cap}_{1}(L)=L$.

В работах [3], [6] доказана

ЛЕмма 1. Для всякой алгебры $L$ сигнатуры $\Omega$ и множсества переменных $x_{1}, x_{2}, \ldots$ существует алгебра $\bar{L}$ той же сигнатуры $\Omega$ такая, что

а) алгебра $L$ является подалгеброй алгебры $\bar{L}$ и алгебра $\bar{L}$ порохсдена алгеброй $L$ и элементами $x_{1}, x_{2}, \ldots$;

б) любое отображсение множества $\left\{x_{i}, i \in \mathbb{N}\right\}$ в алгебру $\bar{L}$ однозначно продолжается до гомоморфизма алгебры $\bar{L}$ в себя, тождественного на $L$

в) элемент $f\left(x_{1}, \ldots, x_{q}\right) \in \bar{L}$, которьй является полиномом сигнатурыл $\Omega$ от $x_{1}, \ldots, x_{q} u$, возмохнно, от әлементов алгебры $L$, равен нулю тогда и только тогда, когда $f\left(v_{1}, \ldots, v_{q}\right)=0$ в L для любих $v_{1}, \ldots, v_{q} \in L$.

Алгебра $\bar{L}$ определена однозначно и является факторалгеброй свободного произведения свободной алгебры сигнатуры $\Omega$ со свободными образующими $x_{1}, x_{2}, \ldots$ и алгебры $L$ по идеалу обобщенных тождеств алгебры $L$.

Очевидно, что множества тождеств алгебры $L$ и $\bar{L}$ совпадают, т.е. $\operatorname{var} L=\operatorname{var} \bar{L}$.

Элементы алгебры $\bar{L}$ можно рассматривать как полиномы сигнатуры $\Omega$ от переменных типа $x: x_{i}, i \in \mathbb{N}$, с коэффициентами из алгебры $L$.

В [6] доказана

ТЕОРема 3. Пусть конечно порохсденная $C$-алгебра $L$ конечной сигнатуры удовлетворяет системе тождеств Капелли порядка $n+1$. Тогда существуют такие коммутативная и ассоциативная $C$-алгебра $\Phi$ с единицей и идеал $J$ Ф-алгебрь $\Phi \otimes_{C} L$, что идеал $J \cap L$ алгебры $L$ нильпотентен 
класса не выше $n$ и в $C$-алгебре $\Phi$ существует такая конечно порожденная $C$-подалгебра $\Phi^{\prime}$, что $\Phi^{\prime}$-модуль $\Phi^{\prime} \cdot L / L \cap J$ конечно порожден.

Там же показано, что в качестве $\Phi$ можно взять кольцо полиномов $\Phi(L)=$ $C\left[\sigma_{q}(a): a \in D(L), 1 \leqslant q \leqslant n\right]$, где $\sigma_{q}(a), a \in D(L), 1 \leqslant q \leqslant n$, являются свободньми коммутирующими переменными. Будем считать, что $\sigma_{0}(a)=1_{C}$ для всех $a \in D(L)$. И там же показано, что в качестве $J$ можно взять $J(L)$ - идеал $\Phi(L)$-алгебры $\Phi(L) \otimes_{C} L$, порожденный элементами вида

$$
\sum_{q=0}^{n}(-1)^{q} \sigma_{q}(a) \otimes a^{n-q} \times v
$$

где $v \in L, a \in D(L)$.

Пусть идеал $W_{n}$ алгебры $\bar{L}$ - это наименьший идеал алгебры $\bar{L}$ среди тех идеалов, которые

1) содержат все элементы вида $d_{n}\left(x_{1}, \ldots, x_{n}\right)$, где $d_{n}\left(x_{1}, \ldots, x_{n}\right)$ - элемент алгебры $\bar{L}$, полилинейный и кососимметричный по переменным $x_{1}, \ldots, x_{n}$ и зависящий, быть может, от других переменных типа $x$;

2) замкнуты относительно всех гомоморфизмов алгебры $\bar{L}$ в себя, которые являются тождественным отображением на $L$ и переводят переменные типа $x$ в переменные типа $x$.

Отметим, что идеал $W_{n}$ содержит все элементы алгебры $\bar{L}$, полилинейные и кососимметричные по $n$ переменным типа $x$, и $W_{n} \subset \mathrm{Cap}_{n}(\bar{L})$.

В [6] доказана

ЛЕмма 2. Пусть $f\left(x_{1}, \ldots, x_{n}, x_{n+1}\right)$ - полином сигнатурь $\Omega$, полилинейный по $x_{1}, \ldots, x_{n}, x_{n+1}$, кососимметричный по $x_{1}, \ldots, x_{n}$ и зависящий, может быть, от других переменных. Тогда полином

$$
f-\left.\sum_{j=1}^{n} f\right|_{x_{j}=x_{n+1}, x_{n+1}=x_{j}}
$$

кососимметричен по $x_{1}, \ldots, x_{n}, x_{n+1}$.

В [7] доказана

ЛЕмма 3. Пусть в алгебре $L$ конечной сигнатуры $\Omega$ над нётеровыц коммутативно-ассоциативным кольцом $C$ выполняются тождества Капелли порядка $n+1$. Тогда $\left(W_{n} . J(\bar{L})\right) \cap \bar{L}=(0)$.

Рассмотрим абсолютно свободную $C$-алгебру $F$ сигнатуры $\Omega$ от свободных образующих типа $x: x_{1}, x_{2}, \ldots$ и типа $z: z_{1}, z_{2}, \ldots$ Пусть сигнатура $\Omega$ содержит операции "арности" не выше $d$.

Пусть $f\left(x_{1}, x_{2}, \ldots, x_{q}, z_{1}, \ldots\right)$ - произвольный моном из алгебры $F$ сигнатуры $\Omega$, полилинейный по $x_{1}, x_{2}, \ldots, x_{q}$ и зависящий, быть может, от переменных типа $z$. Зафиксируем натуральное $s$. Тогда для $q \geqslant d \cdot s$ после перенумерации переменных типа $x$ и типа $z$ моном $f$ имеет вид:

$$
\left.f_{1}\left(x_{1}, \ldots, x_{m}, z_{1}, \ldots\right)\right|_{z_{1}=f_{2}},
$$

где моном $f_{1}$ полилинеен по $x_{1}, \ldots, x_{m}, z_{1}$ и зависит, быть может, от других переменных типа $z$, моном $f_{2}$ полилинеен по $x_{m+1}, \ldots, x_{q}$ и зависит, быть может, от переменных типа $z$, причем $s \leqslant q-m<d \cdot s$ (см. [7]). 


\section{§2. Доказательство теоремы 1}

Для доказательства теоремы 1 достаточно доказать следуюшую лемму, представляюшую самостоятельный интерес.

Лемма 4. Положим $R \stackrel{\text { def }}{=} \operatorname{Cap}_{n}(L) \cap B(L)$. Тогда $R \subseteq B_{1}(L)$.

Для доказательства леммы 4 достаточно доказать следующее утверждение.

Лемма 5. Для любъх $r, t>0$ найдется такое натуральное $l$, ито

$$
R^{l} \subseteq\left(R^{r}\right)^{t}
$$

ДокАЗАТЕЛЬСтво ЛЕммы 5. Зафикиируем $r$. Если $t=1$, то утверждение очевидно и $l(1)=r$. Предположим, что утверждение доказано для некоторого $t$ и

$$
R^{l(t)} \subseteq\left(R^{r}\right)^{t} .
$$

Докажем, что в качестве $l(t+1)$ можно взять $d \cdot l(t)+d \cdot r+1$, где $d$ - максимальная “арность" операций из сигнатуры $\Omega$.

Пусть $f\left(x_{1}, x_{2}, \ldots, x_{l(t+1)}, z_{1}, \ldots\right)$ - произвольный моном из алгебры $F$ сигнатуры $\Omega$, полилинейньй по $x_{1}, x_{2}, \ldots, x_{l(t+1)}$ и зависяший, быть может, от переменных типа $z$. Достаточно доказать, что для любых $v_{1}, \ldots, v_{l(t+1)} \in R$, $w_{1}, w_{2}, \ldots \in L$

$$
\left.f\right|_{x_{1}=v_{1}, \ldots, x_{l(t+1)}=v_{l(t+1)}, z_{1}=w_{1}, z_{2}=w_{2}, \ldots} \in\left(R^{r}\right)^{t+1}
$$

в алгебре $L$.

Тогда моном $f$ после перенумерации переменных типа $x$ и типа $z$ имеет вид:

$$
\left.f_{1}\left(x_{1}, \ldots, x_{m}, z_{1}, \ldots\right)\right|_{z_{1}=f_{2}},
$$

где моном $f_{1}$ полилинеен по $x_{1}, \ldots, x_{m}, z_{1}$ и зависит, быть может, от других переменных типа $z$, а моном $f_{2}$ полилинеен по $x_{m+1}, \ldots, x_{l(t+1)}$ и зависит, быть может, от переменных типа $z$, причем $l(t) \leqslant l(t+1)-m<d \cdot l(t)$. Поэтому для любых $v_{m+1}, \ldots, v_{l(t+1)} \in R, w_{1}, w_{2}, \ldots \in L$

$$
\left.f_{2}\right|_{x_{m+1}=v_{m+1}, \ldots, x_{l(t+1)}}=v_{l(t+1)}, z_{1}=w_{1}, z_{2}=w_{2}, \ldots \in\left(R^{r}\right)^{t}
$$

в алгебре $L$.

Достаточно доказать, что для произвольного монома $g\left(x_{1}, \ldots, x_{m}, z_{1}, \ldots\right)$ из алгебры $F$ сигнатуры $\Omega$, полилинейного по $x_{1}, x_{2}, \ldots, x_{m}, z_{1}$, и зависящего, быть может, от других переменных типа $z$, где $m>d \cdot r+1$, для любых $v_{1}, \ldots, v_{m} \in R$, $w_{1} \in\left(R^{r}\right)^{t}, w_{2}, \ldots \in L$

$$
\left.g\right|_{x_{1}=v_{1}, \ldots, x_{m}=v_{m}, z_{1}=w_{1}, z_{2}=w_{2}, \ldots} \in\left(R^{r}\right)^{t+1}
$$

в алгебре $L$. 
Этот моном $g$ после перенумерации переменных типа $x$ и типа $z$, кроме $z_{1}$, имеет Вид:

$$
\left.g_{1}\left(x_{1}, \ldots, x_{j}, z_{2}, \ldots\right)\right|_{z_{2}=g_{2}},
$$

где моном $g_{1}$ полилинеен по $x_{1}, \ldots, x_{j}, z_{2}$ и зависит, быть может, от других переменных типа $z$, а моном $g_{2}$ полилинеен по $x_{j+1}, \ldots, x_{m}$ и зависит, быть может, от переменных типа $z$, причем $r \leqslant m-j<d \cdot r$. Таким образом, $j \geqslant 1$.

Поэтому для любых $v_{j+1}, \ldots, v_{m} \in R, w_{1}, w_{2}, \ldots \in L$

$$
\left.g_{2}\right|_{x_{j+1}=v_{j+1}, \ldots, x_{m}=v_{m}, z_{1}=w_{1}, z_{2}=w_{2}, \ldots} \in R^{r}
$$

в алгебре $L$.

Если моном $g_{1}$ зависит от $z_{1}$, то для любых $v_{1}, \ldots, v_{m} \in R, w_{1} \in\left(R^{r}\right)^{t}$, $w_{2}, \ldots \in L$

$$
\begin{aligned}
\left.g\right|_{x_{1}} & =v_{1}, \ldots, x_{m}=v_{m}, z_{1}=w_{1}, z_{2}=w_{2}, \ldots \\
& =\left.\left(\left.g_{1}\left(x_{1}, \ldots, x_{j}, z_{1}, z_{2}, \ldots\right)\right|_{z_{2}=g_{2}}\right)\right|_{x_{1}=v_{1}, \ldots, x_{m}=v_{m}, z_{1}=w_{1}, \ldots} \\
& =\left.g_{1}\right|_{x_{1}=v_{1}, \ldots, x_{j}=v_{j}, z_{1}=w_{1}, z_{2}=u, \ldots, z_{i}=w_{i}, \ldots} \in\left(R^{r}\right)^{t+1}
\end{aligned}
$$

в алгебре $L$, поскольку $w_{1} \in\left(R^{r}\right)^{t}$. Так как элемент $u$ лежит в $R^{r}$, утверждение леммы 5 в этом случае доказано.

Если моном $g_{1}$ не зависит от $z_{1}$, тогда моном $g_{2}$ линеен по $z_{1}$. Поскольку $R=\operatorname{Cap}_{n}(L) \cap B(L)$, достаточно доказать, что

$$
\left.g\right|_{x_{1}=v_{1}, \ldots, x_{m}=v_{m}, z_{1}=w_{1}, z_{2}=w_{2}, \ldots} \in\left(R^{r}\right)^{t+1}
$$

в алгебре $L$, если $v_{1} \in \operatorname{Cap}_{n}(L), v_{2}, \ldots, v_{m} \in R, w_{1} \in\left(R^{r}\right)^{t}, w_{2}, \ldots \in L$.

Поэтому достаточно доказать, что для любого полинома $h\left(y_{1}, \ldots, y_{n}, y_{n+1}, \ldots\right)$ сигнатуры $\Omega$ из алгебры $F$, полилинейного и кососимметричного по переменным $y_{1}, \ldots, y_{n}$ типа $z$ и зависящего, быть может, от других переменных типа $z$, полином $\left.g\right|_{x_{1}=h}$ принимает значения из $\left(R^{r}\right)^{t+1}$ при подстановке вместо переменных типа $x$ - элементов из $R$, вместо $z_{1}$ - элемента из $\left(R^{r}\right)^{t}$, а вместо остальных переменных типа $z$-элементов из алгебры $L$.

Поскольку в алгебре $L$ выполняются тождества Капелли порядка $n+1$, то по лемме 2 полином

$$
\left.g\right|_{x_{1}=h}-\left.\sum_{i=1}^{n}\left(\left.g\right|_{x_{1}=h}\right)\right|_{y_{i}=z_{1}, z_{1}=y_{i}}
$$

обращается в нуль в алгебре $L$ при всех подстановках вместо переменных типа $x$ и типа $z$ элементов из алгебры $L$.

Поэтому достаточно доказать, что для всякого $i$ полином $\left.\left(\left.g\right|_{x_{1}=h}\right)\right|_{y_{i}=z_{1}, z_{1}=y_{i}}$ принимает значения из $\left(R^{r}\right)^{t+1}$ при подстановке вместо переменных типа $x$-элементов из $R$, вместо $z_{1}$ - элемента из $\left(R^{r}\right)^{t}$, а вместо остальных переменных типа $z$ - элементов из алгебры $L$. Достаточно доказать этот факт для $i=1$.

Тогда

$$
\begin{gathered}
\bar{h}=\left.h\left(z_{1}, y_{2}, \ldots, y_{n}, \ldots\right)\right|_{z_{1}=w_{1}, y_{2}=w_{2}, \ldots} \in\left(R^{r}\right)^{t} \\
\bar{g}_{2}=\left.g_{2}\right|_{x_{j+1}=v_{j+1}, \ldots, x_{m}=v_{m}, z_{2}=w_{2}, \ldots} \in R^{r}
\end{gathered}
$$


в алгебре $L$ для произвольных $w_{1} \in\left(R^{r}\right)^{t}, w_{2}, \ldots \in L, v_{j+1}, \ldots, v_{m} \in R$.

Тогда элемент

$$
\left.g_{1}\left(x_{1}, \ldots, x_{j}, z_{2}, \ldots\right)\right|_{x_{1}=\bar{h}, x_{2}=v_{2}, \ldots, z_{2}=\bar{g}_{2}, z_{i}=w_{i}, \ldots}
$$

лежит в $\left(R^{r}\right)^{t+1}$, где $\bar{h} \in\left(R^{r}\right)^{t}, \bar{g}_{2} \in R^{r}$, а $v_{i} \in R, w_{i} \in L$. Таким образом, утверждение леммы 5 полностью доказано.

ДоКАЗАТЕЛЬСТво ЛЕммЫ 4. По теореме 2 [5] длина бэровской цепочки идеалов алгебры $L$ конечна, а теорема 1 [7] позволяет утверждать, что найдутся такие натуральњые $r_{1}, \ldots, r_{s}$, что $\left(\ldots\left(\left(B(L)^{r_{1}}\right)^{r_{2}}\right) \ldots\right)^{r_{s}}=(0)$. Тогда $\left(\ldots\left(\left(R^{r_{1}}\right)^{r_{2}}\right) \ldots\right)^{r_{s}}=(0)$. Из леммы 5 получаем, что для достаточно большого $l$ вьполняется равенство $R^{l}=(0)$. Лемма 4 доказана.

ДОКАЗАТЕЛЬСТВо ТЕОРЕМЫ 1 . Проведем индукцию по порядку тождеств Капелли. Пусть алгебра $L$ удовлетворяет тождествам Капелли порядка 2. Тогда $\operatorname{Cap}_{1}(L)=L, B(L)=B(L) \cap \mathrm{Cap}_{1}(L)$. По лемме 4 идеал $B(L)$ нильпотентен, а значит, $B_{1}(L)=B(L)$. Основание индукции доказано.

Пусть во всех конечно порожденных алгебрах $A$ конечной сигнатуры $\Omega$, удовлетворяющих тождествам Капелли порядка $n$, вьполняется $B_{n-1}(A)=B(A)$.

Пусть алгебра $L$ удовлетворяет тождествам Капелли порядка $n+1, n>1$. Тогда $B_{n-1}\left(L / \operatorname{Cap}_{n}(L)\right)=B\left(L / \operatorname{Cap}_{n}(L)\right)$,

$$
B(L) /\left(\operatorname{Cap}_{n}(L) \cap B(L)\right) \subseteq B_{n-1}\left(L / \operatorname{Cap}_{n}(L)\right) .
$$

В силу $\operatorname{Cap}_{n}(L) \cap B(L) \subseteq B_{1}(L)$ получаем $B_{n}(L)=B(L)$. Теорема 1 доказана.

ПримеР. Рассмотрим $n$-мерную бинарную алгебру $L$ с базисом $e_{1}, \ldots, e_{n} . \mathrm{У}_{\mathrm{M}-}$ ножение на базисных элементах задается следующим образом: $e_{i} \cdot e_{i}=e_{i+1}$, если $1 \leqslant i<n ; e_{n} \cdot e_{n}=0, e_{i} \cdot e_{j}=0$, если $i>j ; e_{i} \cdot e_{j}=e_{j}$, если $i<j$. Тогда $B_{i}(L)=\left\langle e_{n-i+1}, \ldots, e_{n}\right\rangle$ для всех $i \in\{1, \ldots, n\}$. Поэтому $B(L)=B_{n}(L) \neq B_{n-1}(L)$. Таким образом, оценка длины бэровской цепочки, даваемая теоремой 1 , не может быть улучшена в классе бинарных алгебр.

\section{§3. Доказательство теоремы 2}

Теорема 2 очевидным образом следует из следующей леммы.

ЛЕмма 6. Пусть $L$ - конечно порожденная алгебра конечной сигнатурь $\Omega$ над полем произвольной характеристики или произвольныцм нётеровым коммутативно-ассочиативныцм кольчом $C$ и $C$-алгебра L удовлетворяет тождествам Капелли порядка $n+1$. Пусть $I_{1}-$ нильпотентный идеал в алгебре L. Тогда в алгебре L существует нильпотентный идеал $I_{2}$ такой, что $I_{1} \subseteq I_{2}$, алгебра $L / I_{2}$ представима и $I_{2}^{n+1} \subseteq I_{1}$.

ДоКАЗАТЕЛЬСтво ЛЕммы 6 . Пусть $R$ - идеал алгебры $L$. Определим идеал $J(L, R)$ как идеал $\Phi(L)$-алгебры $\Phi(L) \otimes_{C} L / R$, порожденный элементами вида

$$
\sum_{q=0}^{n}(-1)^{q} \sigma_{q}(a) \otimes \bar{a}^{n-q} \times v,
$$


где $v \in L / R, a \in D(L), \bar{a}$ - образ а при естественном эпиморфизме из $D(L)$ в $D(L / R)$. Тогда по теореме 3 идеал $J(L, R) \cap L / R$ нильпотентен класса не вьше $n$, и факторалгебра $(L / R) /(J(L, R) \cap L / R)$ представима. Определим $I(L, R)$ как полный прообраз идеала $J(L / R) \cap L / R$ алгебры $L / R$ в алгебре $L$ при естественном гомоморфизме $\varphi: L \rightarrow L / R$. Тогда $R \subseteq I(L, R), I(L, R)^{n+1} \subseteq R$ и алгебра $L / I(L, R)$ представима. Из определения идеала $I(L, R)$ следует, что

$$
I(L, R)=\left(\Phi(L) \otimes_{C} R+J(L)\right) \cap L .
$$

Проведем индукцию по порядку тождеств Капелли. Пусть алгебра $L$ удовлетворяет тождествам Капелли порядка 2. Тогда в качестве $I_{2}$ возьмем $I\left(L, I_{1}\right)$. Из определения идеала $I\left(L, I_{1}\right)$ и теоремы 3 получаем, что $\left(I_{2} . I_{2}\right) \subseteq I_{1}$ и алгебра $L / I_{2}$ представима. Так как идеал $I_{1}$ нильпотентен, получаем $I_{2} \subseteq B_{2}(L)$. Из теоремы 1 следует, что $B_{2}(L)=B_{1}(L)$. По теореме 1 [7] следует, что $B_{1}(L)=N(L)$. Таким образом, идеал $I_{2}$ нильпотентен. Основание индукции доказано.

Пусть лемма 6 верна для всех алгебр, удовлетворяющих тождествам Капелли порядка $n$. Положим $I_{2}=I\left(L, I_{1}\right)$. Тогда $I\left(L, \operatorname{Cap}_{n}(L)+I_{1}\right) \supseteq I\left(L, I_{1}\right)=I_{2}$.

По предположению индукции для некоторого $t$

$$
(\underbrace{I_{2} \ldots I_{2}}_{t \text { раз }}) \subseteq \operatorname{Cap}_{n}(L) .
$$

Пусть выполняется следуюшее:

1) идеал $I_{1}$ алгебры $L$ нильпотентен класса $r$;

2) $f\left(x_{1}, x_{2}, \ldots, x_{q}, z_{1}, \ldots\right)$ - произвольньй моном из свободной алгебры $F$ сигнатуры $\Omega$, полилинейный по $x_{1}, x_{2}, \ldots, x_{q}$ и зависящий, быть может, от переменных типа $z$

3) $q>d \cdot t+r$, где $d$ - максимальная "арность" операций из сигнатуры $\Omega$.

Тогда после перенумерации переменных типа $x$ и типа $z$ полином $f$ имеет вид:

$$
\left.f_{1}\left(x_{1}, \ldots, x_{m}, z_{1}, \ldots\right)\right|_{z_{1}=f_{2}}
$$

где моном $f_{1}$ полилинеен по $x_{1}, \ldots, x_{m}, z_{1}$ и зависит, быть может, от других переменных типа $z$, а моном $f_{2}$ полилинеен по $x_{m+1}, \ldots, x_{q}$ и зависит, быть может, от переменных типа $z$, причем $t \leqslant q-m<d \cdot t$. Поэтому $m>r$.

Подставляя в полином $f$ произвольным образом вместо переменных типа $x$ произвольные элементы из $I_{2}$, а вместо переменных типа $z$ - произвольные элементы из $L$, получаем

$$
\left.f\right|_{x_{1}=v_{1}, \ldots, x_{q}=v_{q}, z_{2}=w_{2}, \ldots}=\left.f_{1}\right|_{x_{1}=v_{1}, \ldots, x_{m}=v_{m}, z_{1}=u, z_{2}=w_{2}, \ldots}
$$

где $v_{1}, \ldots, v_{q} \in I_{2}, w_{2}, \ldots \in L, u \in \operatorname{Cap}_{n}(L)$, поскольку степень полинома $f_{2}$ по переменньм типа $x$ не менее $t$.

Поэтому

$$
(\underbrace{I_{2} \ldots I_{2}}_{q \text { раз }}) \subseteq(\operatorname{Cap}_{n}(L) \cdot \underbrace{I_{2} \ldots I_{2}}_{r+1 \text { раз }}) .
$$


Так же, как и при доказательстве теоремы 1, заметим, что всякий элемент из $\operatorname{Cap}_{n}(L)$ является $C$-линейной комбинацией элементов вида $\left.h\right|_{x_{1}=v_{1}, x_{2}=v_{2}, \ldots}$, где $h$ - элемент из $W_{n}, v_{1}, v_{2}, \ldots \in L$.

Поэтому для доказательства леммы 6 достаточно доказать, что

$$
(W_{n} \cdot \underbrace{\bar{I}_{2} \ldots \bar{I}_{2}}_{r+1 \text { раз }})=(0)
$$

где $\bar{I}_{2}$ - идеал алгебры $\bar{L}$, порожденный идеалом $I_{2}$.

Так как

$$
(\underbrace{I_{1} \ldots . I_{1}}_{r+1 \text { раз }})=(0),
$$

получаем

$$
\begin{aligned}
(W_{n} \cdot \underbrace{\bar{I}_{2} \ldots . \bar{I}_{2}}_{r+1 \text { раз }}) & \subseteq\left(\left(W_{n} . J(\bar{L})\right)+\Phi(\bar{L}) \otimes_{C}(W_{n} \cdot \underbrace{\bar{I}_{1} \ldots \bar{I}_{1}}_{r+1 \text { раз }})\right) \cap \bar{L} \\
& \subseteq\left(W_{n} . J(\bar{L})\right) \cap \bar{L} .
\end{aligned}
$$

По лемме 3 имеем

$$
\left(W_{n} . J(\bar{L})\right) \cap \bar{L}=(0) .
$$

Таким образом, идеал $I_{2}$ нильпотентен класса не выше $q$.

Доказательство леммы 6 окончено.

\section{Список литературы}

1. Baer R. Radical ideals // Amer. J. Math. 1943. V. 65. P. 537-568.

2. Размыслов Ю. П. О радикале Джекобсона в РІ-алгебрах // Алгебра и логика. 1974. T. 13. № 3. C. 337-360.

3. Размыслов Ю. П. Алгебры, удовлетворяющие тождественным соотношениям типа Капелли // Изв. АН СССР. Сер. матем. 1981. Т. 45. № 1. С. 143-166.

4. Razmyslov Yu. P., Zubrilin K. A. Capelli identities and representations of finite type // Comm. Algebra. 1994. V. 22. № 14. P. 5733-5744.

5. Зубрилин K. А. Алгебры, удовлетворяющие тождествам Капелли // Матем. сб. 1995. T. 186. № 3. C. 53-64.

6. Зубрилин K.A. О классе нильпотентности препятствия для представимости алгебр, удовлетворяющих тождествам Капелли // Фундамент. и прикл. матем. 1995. Т. 1. № 2. C. $409-430$.

7. Зубрилин K. А. О наибольшем нильпотентном идеале в алгебрах, удовлетворяющих тождествам Капелли // Матем. сб. 1997. Т. 188. № 8. С. 93-102.

8. Кемер А. Р. Тождества Капелли и нильпотентность радикала Джекобсона конечно порожденной РІ-алгебры // Докл. АН СССР. 1980. Т. 245. № 4. С. 793-799.

9. Braun A. The Jacobson radical in a finitely generated P.I. algebra // Bull. Amer. Math. Soc. 1982. V. 7. № 2. P. 385-386.

10. Kaplansky I. Rings with polynomial identity // Bull. Amer. Math. Soc. 1948. V. 54. P. 575-589. 